SLAC-PUB- 755

June 1970

(TH) \& (EXP)

\title{
MASSIVE LEPTON PAIR PRODUCTION IN HADRON-HADRON COLLISIONS
}

\author{
AT HIGH ENERGIES*
}

Sidney D. Drell and Tung-Mow Yan

Stanford Linear Accelerator Center

Stanford University, Stanford, California 94305

\begin{abstract}
On the basis of a parton model studied earlier we consider the production process of large mass lepton pairs from hadron-hadron inelastic collisions in the limiting region, $s \rightarrow \infty, Q^{2} / s$ finite, $Q^{2}$ and $s$ being the squared invariant masses of the lepton pair and the two initial hadrons, respectively. General scaling properties and connections with deep inelastic electron scattering are discussed. In particular, a rapidly decreasing cross section as $Q^{2} / s \rightarrow 1$ is predicted as a consequence of the observed rapid fall-off of the inelastic scattering structure function $\nu \mathrm{W}_{2}$ near threshold。
\end{abstract}

(Submitted to Phys. Rev. Letters)

\footnotetext{
Work supported by the U。S.Atomic Energy Commission.
} 
Feynman's parton model ${ }^{1}$ for deep inelastic weak or electromagnetic processes is an expression of the impulse approximation as applicd to elementary particle interactions. In order to apply the impulse approximation we demand the following. We analyze the bound system - be it a nucleon or nucleus - in terms of its constitutents, called "partons." Nucleons are the "partons" of the nucleus and the "partons" of a nucleon itself are still to be deciphered. If we specify the kinematics so that the partons can be treated as instantaneously free during the sudden pulse carrying the large energy transfer from the projectile (or lepton) then we can neglect their binding effects during the interaction and we can treat the kinematics of the collision as between two free particles - the projectile and the parton. Moreover if we are in a kinematic regime so that energy is approximately conserved along with momentum across the interaction vertex of the parton with the weak or electromagnetic current, the conditions for applying the impulse approximation are satisfied.

The Bjorken limiting region ${ }^{2}$ satisfies this condition for the deep inelastic electron scattering from protons as viewed from a certain class of $\mathrm{P} \rightarrow \infty$, or infinite momentum frames. The "partons" constituting a proton are strongly bound together as viewed in the rest frame. However if their bound state can be formed primarily by momentum components that are limited in magnitude below some fixed maximum - i.e., if there exists a finite $\mathrm{k}_{\max }$ - then as viewed in an infinite momentum frame these parton states are long lived by virture of the characteristic time dilation. The derivation of this intuitively appealing picture from a canonical quantum field, modified by imposing a maximum constraint on $\mathrm{k}_{1}$, has been discussed as well as its applicability to the particular class of amplitudes with "good currents. " In particular, the ratio $Q^{2} / 2 M \nu$, where $Q^{2}>0$ is the negative of the square of the invariant momentum transfer and $q \cdot \mathrm{P}=\mathrm{M} \nu$, measures the fraction $\mathrm{x} \equiv \mathrm{Q}^{2} / 2 \mathrm{M} \nu$ of the longitudinal momentum on the parton from which the electron scatters and is a finite fraction $0<x<1$ in the Bjorken limit:

It is easy to show that the ratio $\mathrm{x}$ must be finite in order to apply the impulse approximation. Otherwise as $\mathrm{x}$ approaches very close to 0 or 1 we will be forced to deal with very 
slow partons in the $\mathrm{P} \rightarrow \infty$ system, or, as seen in the rest system of the proton, with the high momentum extremities of the bound state structure, and for these the impulse approximation breaks down.

The beauty of the electron scattering is that it allows us to "tune" the mass of the virtual photon line as we choose to probe finite $\mathrm{x}$. However when we return to the world of only real external hadrons, we have no large mass since $Q^{2} \rightarrow M^{2}$ while $2 M \nu \rightarrow s$ the total collision energy. In this case $\mathrm{x}$ becomes very small ${ }^{1}$ - or "wee." Our condition for applying the impulse approximation also fails and the value of the parton concept is less certain. ${ }^{4}$ The impulse approximation also applies to electron-positron pair annihilation into a specific hadron $\mathrm{H}$ plus anything else: $\mathrm{e}^{+}+\mathrm{e}^{-} \rightarrow \mathrm{H}+$ "anything" in the deep inelastic region of large lepton pair mass squared ${ }^{2}$ and large invariant energy transfer $\nu$. In an infinite momentum frame of the detected hadron, this process can be described as the creation of an essentially free parton-anti-parton pair and its subsequent decay into final states.

If we want to find other processes which satisfy the kinematical constraints allowing application of the impulse approximation we need look for interactions at high energies $\mathrm{s}$ which absorb or produce a lepton system of huge mass $Q^{2}$ such that the ratio $Q^{2} / s$ is finite. An observable class of processes meeting this requirement is production of massive lepton pairs in hadron-hadron collisions, ${ }^{5}$ viz

$$
\mathrm{p}+\mathrm{p}-\left(\mu^{+} \mu^{-}\right)+\ldots
$$

Our remarks apply equally to any colliding pair such as (pp), (pp), ( $\pi p),(\gamma p)$ and to final leptons $\left(\mu^{+} \mu^{-}\right),(\overline{\mathrm{e}}),(\mu \nu)$, and $(\mathrm{e} \nu)$.

What is going on here can be best illustrated in a center-of-mass frame. If a massive state with $Q^{2} \sim s$ emerges from one of the colliding protons (A) or (B) as in $\mathrm{Fig}$. 1a, it is impossible to satisfy both energy and momentum conservation in the overall collision and at the same time exchange only "wee" partons between (A) and (B). ${ }^{6}$ Hence this process will not be related directly to the total nucleon-nucleon cross section ${ }^{7}$ in which, as discussed by Feynman, it is the "wee" partons with $x-1 \mathrm{GeV} / \sqrt{\mathrm{s}}$ that can't tell "right" 
from "left" in Fig. 1a that are responsible for $\sigma_{\mathrm{T}}$. In contrast the dominant amplitude in (1) in a model of the nucleon with a finite momentum $\mathrm{k}_{\max }$ in its ground state structure will be the production of the massive lepton pair by annihilation of an anti-parton-parton pair as illustrated in Fig. 1b. Viewed from the center-of-mass frame a hard (i. e., non"wee") parton moving to the right, say, annihilates on a similar antiparton headed to the left and the resulting system is very massive since their energies add wheras their momenta subtract. It is easy to show that if a pair of mass $Q^{2}$ is formed

$$
\mathrm{Q}^{2}=\mathrm{x}_{1} \mathrm{x}_{2} \mathrm{~s} ; \quad 0<\mathrm{x}_{1,2}<1
$$

where $x_{1,2}$ are the fractions of the longitudinal momenta of their respective hadrons carried by the annihilating parton pair. Clearly for finite $\mathrm{Q}^{2} / \mathrm{s}$ one is here dealing with hard partons and with the same region of momenta as probed by deep inelastic scattering experiments which measure the parton distribution in $\mathrm{x}=\mathrm{Q}^{2} / 2 \mathrm{M} \nu$. In this process we are measuring over a range of their values as constrained by (2) for fixed $Q^{2} / \mathrm{s}$.

We now turn to a calculation of (1) in the deep inelastic region of finite $\tau=Q^{2} / \mathrm{s}$ with $\mathbf{s} \rightarrow \infty$. The general expression for the cross section is $\frac{\mathrm{d} \sigma}{\mathrm{dQ}^{2}}=\left(\frac{4 \pi \alpha^{2}}{3 \mathrm{Q}^{2}}\right) \sqrt{1-\frac{4 \mathrm{~m}^{2}}{\mathrm{Q}^{2}}}\left(1+\frac{2 \mathrm{~m}^{2}}{\mathrm{Q}^{2}}\right) \frac{1}{\left.\left.\sqrt{\left[\mathrm{s}-\left(\mathrm{M}_{1}+\mathrm{M}_{2}\right)\right.}\right)^{2}\right]\left[\mathrm{s}-\left(\mathrm{M}_{1}-\mathrm{M}_{2}\right)^{2}\right]} W\left(\mathrm{Q}^{2}, \mathrm{~s}\right)$ where a spin average is understood and

$$
\begin{aligned}
W\left(Q^{2}, \mathrm{~s}\right) \equiv & -16 \pi^{2} E_{1} E_{2} \int(\mathrm{dq}) \delta\left(\mathrm{q}^{2}-\mathrm{Q}^{2}\right) \int(\mathrm{dx}) \mathrm{e}^{-\mathrm{iqx}}\left\langle\mathrm{P}_{1} \mathrm{P}_{2}^{(\mathrm{in})}\left|\mathrm{J}_{\mu}(\mathrm{x}) J^{\mu}(0)\right| \mathrm{P}_{2} \mathrm{P}_{1}^{(\mathrm{in})}\right\rangle \\
=- & 16 \pi^{2} \mathrm{E}_{1} \mathrm{E}_{2} \int(\mathrm{dq}) \delta\left(\mathrm{q}^{2}-\mathrm{Q}^{2}\right) \sum_{\mathrm{n}}(2 \pi)^{4} \delta^{4}\left(\mathrm{P}_{1}+\mathrm{P}_{2}-\mathrm{q}-\mathrm{P}_{\mathrm{n}}\right) \times \\
& \times\left\langle\mathrm{P}_{1} \mathrm{P}_{2}^{(\mathrm{in})}\left|\mathrm{J}_{\mu}\right| \mathrm{n}\right\rangle\left\langle\mathrm{n}\left|J^{\mu}\right| \mathrm{P}_{2} \mathrm{P}_{1}^{(\mathrm{in})}\right\rangle .
\end{aligned}
$$

In (4) $\mathrm{E}_{1}, \mathrm{P}_{1}, \mathrm{M}_{1}$ and $\mathrm{E}_{2}, \mathrm{P}_{2}, \mathrm{M}_{2}$ are the energies, momenta, and masses of the two initial hadrons and $m$ is the muon mass. Since we will directly imitate the steps in our preceding analyses of deep inelastic processes ${ }^{3}$ we first define a true infinite momentum frame by boosting from the collision center-of-mass frame by a velocity $\beta / \sqrt{1-\beta^{2}}=2 \mathrm{P} / \sqrt{\mathrm{s}}$ 
in a direction orthogonal to the collision axis. The four-vector momenta of the two incident colliding hadrons are then, for $\mathrm{s} \gg>\mathrm{M}^{2}$

$$
\begin{aligned}
& \mathrm{P}_{1}^{\mu}=\left(\mathrm{P}+\frac{\mathrm{s}}{8 \mathrm{P}}, \frac{1}{2} \sqrt{\mathrm{s}}, 0, \mathrm{P}\right), \\
& \mathrm{P}_{2}^{\mu}=\left(\mathrm{P}+\frac{\mathrm{s}}{8 \mathrm{P}},-\frac{1}{2} \sqrt{\mathrm{s},} 0, \mathrm{P}\right) .
\end{aligned}
$$

We can now let $\mathrm{P} \rightarrow \infty$ for large but finite $\mathrm{s}: \mathrm{P} \gg \sqrt{\mathrm{S}} \gg \mathrm{M}$. The energy in the collision is represented by a transverse momentum mismatch of the colliding hadrons. For a parton, or a baryon or meson quantum in our field theory model, to be exchanged between them without introducing an asymptotically large momentum transverse to either of the two hadron lines, the parton momentum is restricted to a fraction $-M / \sqrt{\mathrm{S}}$ along the $\mathrm{P}$ axis and to a finite value $-M$ orthogonal to it. This constraint corresponds to the "wee" parton condition in the center-of-mass frame of the colliding hadrons. In the P $\rightarrow \infty$ frame (5) this constraint satisfies the condition of finite transverse momentum imposed on our field theory model.

In this frame $e^{8}$ we can repeat steps developed in earlier work of undressing the current operator by the U matrix: $J_{\mu}(0)=U^{-1} j_{\mu}(0) U$ where $j_{\mu}(0)$ is the current operator expressed in terms of free fields. Furthermore the energy differences between the eigenstate $\left|P_{1} P_{2}^{(i n)}\right\rangle$ and the components of $U\left|P_{1} P_{2}^{(\text {in) }}\right\rangle$ can be ignored in the limit $s \rightarrow \infty$ for $Q^{2} / s$ finite; the same is true for $|n\rangle$ and $U|n\rangle$. This is so because the invariant mass of the individual system of particles moving along $\underset{\sim}{\mathrm{P}}$ and $\mathrm{P}_{2}$ respectively in (5), or to the right and left in the center-of-mass frame, is finite as a result of the transverse momentum cutoff imposed. This mass is thus negligible compared with the invariant mass $1 / 2 \sqrt{\mathrm{s}}$ appearing in (5). In other words the impulse approximation is good and energy as well as momentum is conserved across the electromagnetic current vertex in (4). This leads to the simplification of (4) in the $\operatorname{Lim}_{b j}$ for $P \rightarrow \infty ; s \gg M^{2} ; Q^{2} / s$ finite to

$$
\operatorname{Lim}_{b j} W=-16 \pi^{2} E_{1} E_{2} \int(d q) \delta\left(q^{2}-Q^{2}\right) \int(d x) e^{-i q x}\left\langle U\left(P_{1} P_{2}\right)^{i n}\left|j_{\mu}(x) j^{\mu}(0)\right| U\left(P_{2} P_{1}\right)^{i n}\right\rangle
$$


and in our model, as described in earlier work, to a factorization of the U matrix

$$
\left|U\left(P_{1} P_{2}\right)^{\text {in }}\right\rangle=\left|U P_{1}\right\rangle\left|U_{2}\right\rangle
$$

Proceeding in analogy with II, Eqs. $72-78$ we find for the annihilation of a boson pair (the same result obtains for a fermion pair with spin averaging)

$$
\begin{aligned}
(-) \int(\mathrm{dq}) & \delta\left(\mathrm{q}^{2}-\mathrm{Q}^{2}\right) \int(\mathrm{dx}) \mathrm{e}^{-\mathrm{iqx}}\left\langle\mathrm{k}_{1} \mathrm{k}_{2}\left|\mathrm{j}^{\mu}(\mathrm{x}) \mathrm{j}_{\mu}(0)\right| \mathrm{k}_{2} \mathrm{k}_{1}\right\rangle \\
& =(2 \pi)^{4} \delta\left[\mathrm{Q}^{2}-\left(\mathrm{k}_{1}+\mathrm{k}_{2}\right)^{2}\right] \frac{-\lambda^{2}}{(2 \pi)^{6}\left(2 \omega_{1}\right)\left(2 \omega_{2}\right)}\left(\mathrm{k}_{1}-\mathrm{k}_{2}\right)_{\mu}\left(\mathrm{k}_{1}-\mathrm{k}_{2}\right)^{\mu} \\
& =\frac{\lambda^{2}}{16 \pi^{2} \mathrm{E}_{1} \mathrm{E}_{2}} \delta\left(\mathrm{x}_{1} \mathrm{x}_{2}-\tau\right) ; \quad \tau \equiv \mathrm{Q}^{2} / \mathrm{s}<1
\end{aligned}
$$

where $\lambda^{2}$ is the square of the charge of an individual parton and we have used the high energy approximation for the dominant large components of the momenta $\mathrm{k}_{1}^{\mu}=\mathrm{x}_{1} \mathrm{P}_{1}^{\mu}, \mathrm{k}_{2}^{\mu}=\mathrm{x}_{2} \mathrm{P}_{2}^{\mu}$. Inserting the identity

$$
1=\int_{0}^{1} \mathrm{~d}\left(\frac{1}{\omega_{1}}\right) \int_{0}^{1} \mathrm{~d}\left(\frac{1}{\omega_{2}}\right) \delta\left(\mathrm{x}_{1}-\frac{1}{\omega_{1}}\right) \delta\left(\mathrm{x}_{2}-\frac{1}{\omega_{2}}\right)
$$

we have

$$
\begin{aligned}
\operatorname{Lim}_{b j} W\left(Q^{2}, s\right) \equiv & \frac{1}{\tau} \mathscr{F}(\tau)=\sum_{a} \lambda_{a}^{2} \int_{0}^{1} \mathrm{~d}\left(\frac{1}{\omega_{1}}\right) \int_{0}^{1} \mathrm{~d}\left(\frac{1}{\omega_{2}}\right) \delta\left(\frac{1}{\omega_{1} \omega_{2}}-\tau\right) \times \\
& \left\langle\mathrm{UP}_{1}\left|\delta\left(\mathrm{x}_{1, \mathrm{a}}-\frac{1}{\omega_{1}}\right)\right| U P_{1}\right\rangle\left\langle U P_{2}\left|\delta\left(\mathrm{x}_{2, \overline{\mathrm{a}}}-\frac{1}{\omega_{2}}\right)\right| U P_{2}\right\rangle
\end{aligned}
$$

where the summation over types of partons with charges $\lambda_{a}$ pairs a parton of type $\mathrm{a}$ in $\left|U P_{1}\right\rangle$ with its anti-parton $\bar{a}$ in $\left|U P_{2}\right\rangle$ and vice versa. By comparison with $(78),(79)$, and $(80)$ of $I I$ we see that $(8)$ can be rewritten as

$$
\mathscr{F}(\tau)=\sum_{\mathrm{a}}\left(\lambda_{\mathrm{a}}\right)^{-2} \int_{1}^{\infty} \mathrm{d} \omega_{1} \int_{1}^{\infty} \mathrm{d} \omega_{2} \delta\left(\omega_{1} \omega_{2}-\frac{1}{\tau}\right) \mathrm{F}_{2 \mathrm{a}}\left(\omega_{1}\right) \mathrm{F}_{2 \overline{\mathrm{a}}}^{\prime}\left(\omega_{2}\right)
$$

in terms of the invariant structure functions $\mathrm{F}_{2 \mathrm{a}}\left(\omega_{1}\right)=\nu_{1} \mathrm{~W}_{2}^{\left(\lambda_{\mathrm{a}}\right)}$ introduced in the deep inelastic scattering analyses (see (78) of II) for $\omega_{1}$ times the probability of finding parton of type a in the proton (or hadron $A$ ) with a momentum fraction $x_{1}=1 / \omega_{1} . F_{2 \pi}^{\prime}\left(\omega_{2}\right)$ has the same significance for the corresponding anti-parton distribution in hadron (B). 
The differential cross section (3) now assumes the simple form in the scaling limit

$$
\frac{\mathrm{d} \sigma}{\mathrm{dQ}^{2}}=\left(\frac{4 \pi \alpha^{2}}{3 \mathrm{Q}^{2}}\right)\left(\frac{1}{\mathrm{Q}^{2}}\right) \pi(\tau)=\left(\frac{4 \pi \alpha^{2}}{3 \mathrm{Q}^{2}}\right)\left(\frac{1}{\mathrm{Q}^{2}}\right) \int_{0}^{1} \mathrm{dx} \int_{0}^{1} \mathrm{dx} \mathrm{x}_{2} \delta\left(\mathrm{x}_{1} \mathrm{x}_{2}-\tau\right) \sum_{\mathrm{a}} \lambda_{\mathrm{a}}^{-2} \mathrm{~F}_{2 \mathrm{a}}\left(\mathrm{x}_{1}\right) \mathrm{F}_{2 \overline{\mathrm{a}}}^{\prime}\left(\mathrm{x}_{2}\right)
$$

where we have rewritten the invariant structure functions in terms of momentum fraction $\mathrm{x} .4 \pi \alpha^{2} / 3 \mathrm{Q}^{2}$ is just the total cross section for e- annihilation into (point) muon pairs in the relativistic limit.

Equation (10) is the central result of this letter and is a formal expression of our earlier discussion. We conclude with several remarks about general features of this result:

1. The observed ${ }^{9}$ rapid decrease of the inelastic structure functions $\mathrm{F}_{2}(\mathrm{x})=\nu \mathrm{W}_{2}$ as $\mathrm{x} \rightarrow 1$ leads in (2) and $(10)$ to a prediction of a very rapid falloff in $\mathscr{F}(\tau)$ with increasing $\tau=Q^{2} / \mathrm{s}$. If we assume that the parton and anti-parton have identical momentum distributions in the proton and this is common for all parton types $\lambda$, we can compute $d \sigma / \mathrm{dQ}^{2}$ directly from measured $\mathrm{F}_{2}(\mathrm{x})$, finding a very rapid falloff in the cross section as shown in Fig. 2, even though the model consists of point-like constituents. This is in qualitative accord with preliminary experimental findings. 5

2. The angular distribution of the vector $q \equiv p_{+}+p_{-}$, the total momentum of the muon pair, is peaked along the incident nucleon's direction in the lab system. This follows from the observation that $q \cdot P_{1}=\left(x_{1} P_{1}+x_{2} P_{2}\right) \cdot P_{1} \equiv \frac{1}{2} x_{2} s$ is an invariant and in terms of laboratory variables $q \cdot P_{1} \cong E_{1} q^{o}(1-\cos \theta)$, with $\mathrm{M}_{2} \mathrm{E}_{1} \cong \frac{1}{2} \mathrm{~s}$, so that $1-\cos \theta \sim 0\left(1 / \mathrm{q}^{\circ}\right)$.

3. The virtual photon will be predominantly transversely polarized if it is formed by annihilation of spin $1 / 2$ parton-antiparton pairs. This means a distribution in the di-muon rest system varying as $\left(1+\cos ^{2} \theta\right)$ rather than $\sin ^{2} \theta$ as found in Sakurai's ${ }^{10}$ vector dominance model where $\theta$ is the angle of the muon with respect to the timelike photon momentum. The model used in Fig. 2 assumed identical partonantiparton distributions and hence the spin $1 / 2$ partons play the predominant role as in the scattering experiments. 9 
4. The full range of processes of the type (1) with incident $p, \vec{p}, \pi, K, \gamma$, etc. , affords the interesting possibility of comparing their parton and anti-parton structures. [In particular no relation between the parton and anti-parton spectra need be assumed, as we did in Fig. 2, for an initial $\bar{p}$ p state.] Not only are the variations important but so are the cross section magnitudes as measures of effective $\lambda^{\prime} s$.

5. The factoring in (7) is possible only because "wee" parton exchanges are absent in our model for processes with hard partons to which an impulse approximation applies. This would not be the case if our theoretical model were enlarged to include a "wee" region of prominence (perhaps due to neutral vector exchanges). Presumably such quanta are needed to generate Feynman's spectrum of "wee" or infrared quanta, dx/x for explaining real hadron cross sections. ${ }^{11}$ Since the impulse condition does not apply in these interactions we cannot compute purely hadronic processes by our techniques as in (6). However we can ask what implications there will be for our results for massive lepton pair production if such "wee" quanta are introduced and modify (7) by initial state interactions.

For example, suppose we include the "wee" parton exchanges between the two systems (A) and (B) before or after the parton-anti-parton annihilation takes place. Precisely because the transfer red momenta are "wee, " these interactions can change the invariant mass of individual groups (A) and (B) in Fig. 1 only by finite amount and the fractions of their longitudinal momenta by order of $1 \mathrm{GeV} / \sqrt{\mathrm{s}}$. These corrections therefore do not affect our arguments leading to (6) which in turn implies (2) and the general scaling (9). Therefore although the invariant function $\pi(\tau)$ will be modified from (9) by the "wee" exchanges, the general scaling property will not be affected. Based on this observation we would like to emphasize that although "wee" exchanges must survive at infinite energies to account for a nonvanishing total cross section of hadron-hadron collisions, they are not relevant to the Bjorken scaling behavior of deep inelastic lepton processes such as electron scattering, electron-positron annihilation and the massive muon pair production 
in proton-proton scattering considered here. A nontrivial Bjorken scaling behavior and the validity of the impulse approximation for these processes are independent of whether or not the total cross section for hadrons vanishes at high energies.

\section{REFERENCES}

1. R.B. Feynman, Phys. Rev. Letters 23,1415 (1969), and unpublished talks.

2. J.D. Bjorken, Phys. Rev. 179, 1547 (1969).

3. S. D. Drell, D.J. Levy, R.M. Yan, Phys. Rev. Letters 22, 744 (1969); Phys. Rev. 187, 2159 (1969); SLAC-PUB-645, -685 (1969)(Phys. Rev., to be published), and T.M. Yan, S. D. Drell, SLAC-PUB-692 (1969) (Phys. Rev., to be published). The last four papers will be referred to as Papers I, II, III, and IV, respectively.

4. However, as suggested by Feynman, we may hope for clues to the behavior here by studying the deep inelastic electron scattering as $\mathrm{x}$ decreases to very small values and thus be led to insights into what is going on here in the "wee" region.

5. L.M. Lederman, Proceedings of the International Symposium on Electron and Photon Interactions at High Energies, Liverpool, England(1969). We Thank Dr. Lederman for discussions of his experiment and its preliminary analysis.

6. Simple calculation shows that the exchanged longitudinal momentum fraction is finite and given by $1 / 2\left[\sqrt{\eta^{2}+4 Q^{2} / s}-\eta\right]$, with $\eta$ being the fractional longitudinal momentum of the virtual photon.

7. For a different interpretation see S. Berman, D. Levy, and T. Neff, Phys. Rev. Letters 23,1363 (1969). For another approach using current commutators see R. F. Kogerler and R. M. Muradyan; Dubna preprint E2-4791 (1969). For a different application to photoproduction with a large momentum transfer as opposed to a large mass $Q^{2}$ see J. Bjorken and E. Paschos, Phys. Rev. 185,1975 (1969).

8. The following discussion also applies if we work in the center-of-mass frame of the two initial hadrons. 
9. E. Bloom et al., Phys. Rev. Letters 23, 930 (1969); M. Breidenbach et al., ibid, 23, 935 (1969); R. Taylor, Invited talk at Daresbury Conference, SLACPUB-677 (1969).

10. J. J. Sakurai, Phys. Rev. Letters 24, 968 (1970).

11. We thank Dr. Ken Wilson for questions and stimulating discussions on this point.

\section{FIGURE CAPTIONS}

1. (a)--Production of a massive pair $\mathrm{Q}^{2}$ from one of the hadrons in a high energy collision. In this case it is kinematically impossible to exchange "wee" partons only. (b)--Production of a massive pair by parton anti-parton annililation.

2. $\frac{\mathrm{d} \sigma}{\mathrm{dQ}^{2}}$ computed from Eq. (10) assuming identical parton and anti-parton momentum distributions and with relative normalization. 


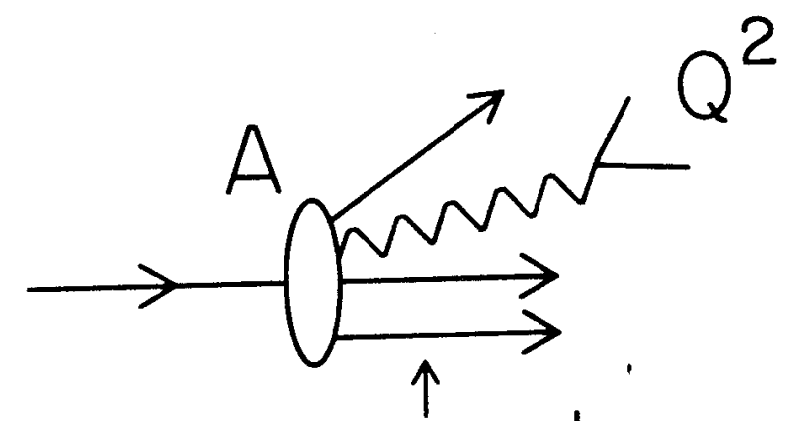
can not be wee only $\downarrow$

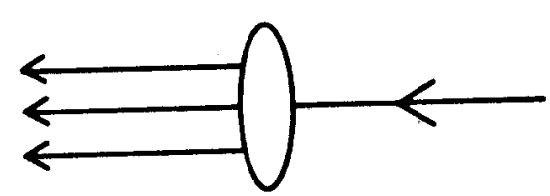

B

1589Al

(a)

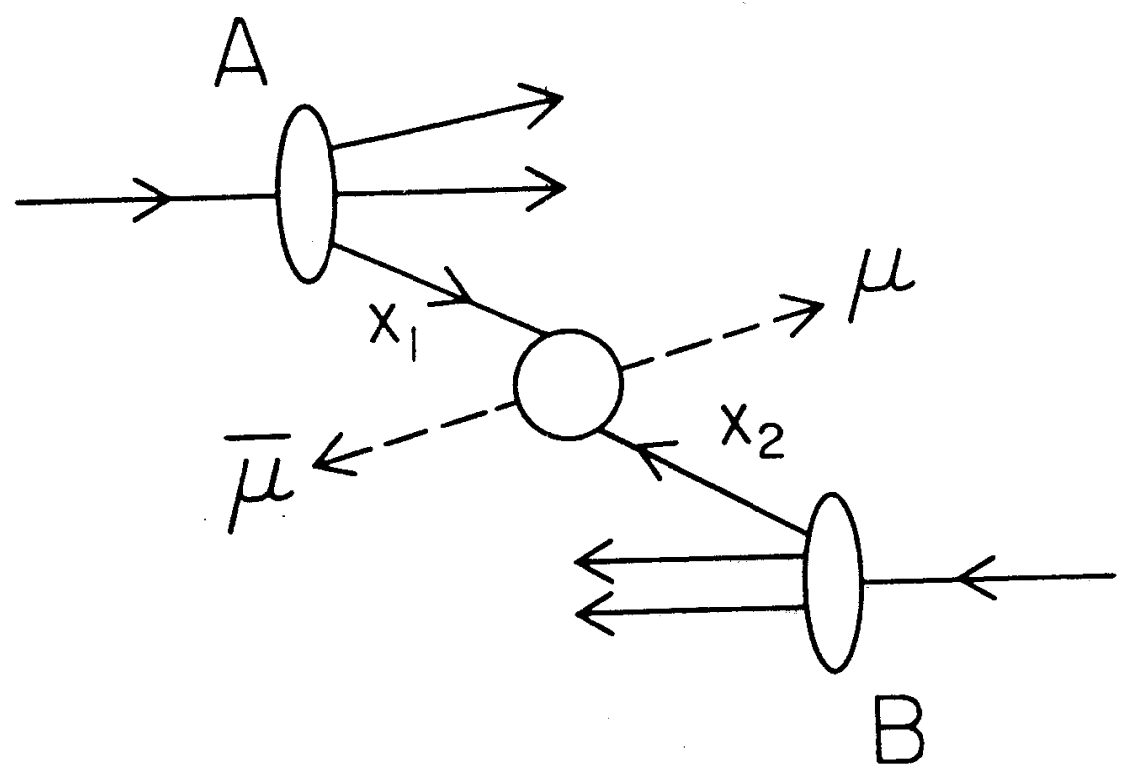

$Q^{2}=x_{1} x_{2} S$

(b)

Fig. 1 


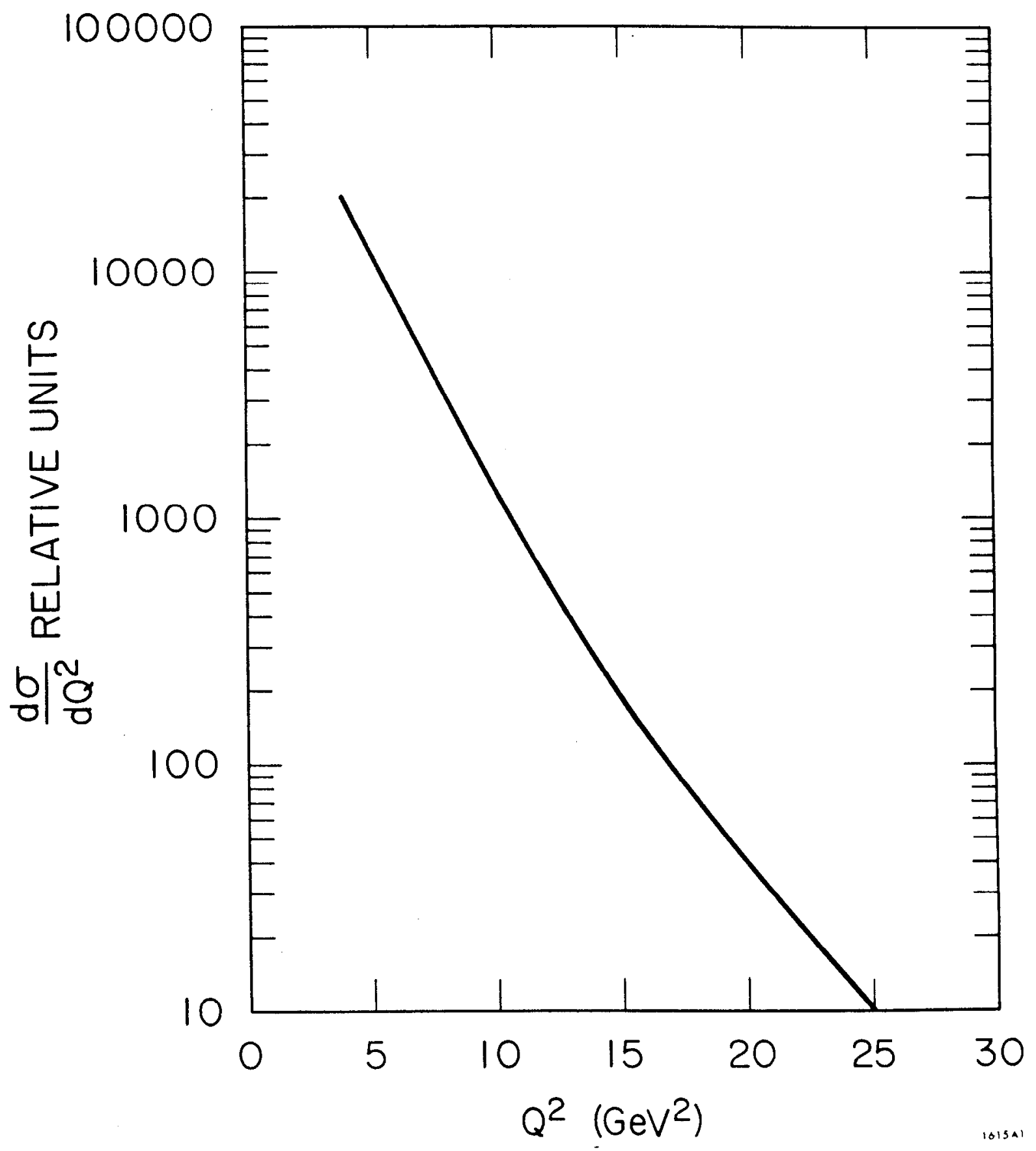

Fig. 2 